\title{
Beyond Drug Regulation: The Science of Drug Evaluation
}

\author{
Alexander Fleming, M.D. ${ }^{1}$ \\ Food and Drug Administration, Rockville, Maryland, U.S.A.
}

These are the best of times and the worst of times for those involved in the discovery, development, and evaluation of medical therapies. The revolutions in molecular biology, electronic technology, global economics, and the political process offer both promise and peril for all scientists wherever and however they pursue their disciplines. Scientists within the U.S. Food and Drug Administration (FDA) have not been shielded from these cataclysms of dangerous opportunity. Despite the uncertainty about the future roles of the FDA itself, there has never been as much intellectual activity within the Agency directed at understanding the principles and improving the processes by which drugs and other therapies are assessed and approved. Within the Agency, there is growing recognition of the scientific discipline involved in the evaluation of drugs. More science and less art in the evaluation of drug research should be welcomed by everyone working to discover and develop new treatments. Support and involvement of those from every area of biomedical science is needed now for the development of this discipline to proceed apace.

Drug evaluation science is more than just the specialized application to the drug development process of well-defined scientific disciplines such as biostatistics, pharmacology, and molecular biology. Membership in one of these or the many other disciplines involved in drug development is necessary but, by itself, insufficient to qualify as a drug evaluation scientist. In essence, the evaluator scientist begins with a detailed understanding of a complex, multidisciplinary process. The practice of the science centers around incessant hypothesis generation and assumption testing. Finally, the logic of and the conclusions reached

\footnotetext{
${ }^{1}$ These are the views of the author and do not necessarily represent the official policies of the Food and Drug Administration.
}

by an evaluator scientist must be clear and compelling to the expert community.

It is useful to understand the crucible from whence the drug evaluation science concept has originated. First, the FDA, like every other federal agency, has been affected by the sea change in political and public sentiment towards government. The prevailing view is that government has become too large, too intrusive, too costly, and too unaccountable for its results. The FDA still enjoys considerable support among the general public for its role as a protector of individuals against large institutional interests. At the same time, the FDA is held increasingly responsible for the availability of treatments and not just for their safety and effectiveness.

Fortunately, the FDA is now able to add professionals and other resources to new drug review because of a user fee system that was approved by Congress in 1992. This arrangement permits the Center for Drug Evaluation and Research (CDER) and its sister, the Center for Biologics Evaluation and Research (CBER), to apply these resources to the review process, allowing them to conduct their work at a much faster pace than has been the case in the past. To date, this agreement has been quite successful in speeding up the drug approval process. The success is due not so much to applying additional resources, but to the fact that most everyone in the Agency, starting with Commissioner David Kessler, knew that it had to be done. This period of increased support should, however, be seen only as a window of opportunity for the Agency to get the personnel, the processes, and the tools to do its job in the 21 st century. Indeed, like nearly all other parts of government, the FDA is already experiencing reduced funding in areas not involved with new drug review. It is only a matter of time before drug review itself will face resource cutbacks. 
Recognition that faster drug review involves much attention to both process and personnel is an important impetus for the FDA's involvement in drug regulatory science and the closely related concept of Good Review Practice (GRP). The FDA will only be able to evaluate drugs well if the principles of drug evaluation are defined, understood, and practiced by Agency professionals and are widely accepted and understood by all those who submit data to the Agency.

The concept of drug evaluation science will also help the Agency respond to the demand that government must be more accountable for its results and not just its outputs. The distinction between outputs and results was put into law with the bipartisan support and passage of the Government Performance and Results Act in 1992. Very simply, in the context of drug review, examples of Agency outputs would include reviews produced, meetings held, and decisions made. These outputs may lead to benefits but, in themselves, are not actual benefits, like mortality reduction or fewer adverse drug reactions. The Agency's accountability for the speed and productivity of therapeutics development is now about equal to that of the developers themselves. While this expectation may overestimate the FDA's importance in the development of new drugs and other therapies, the FDA's adjudged performance in this arena hinges again on a streamlined, efficient process understood by reviewers and developers alike. Drug evaluation science will support the expeditious development of needed therapies and other recognizable results.

The concept of drug evaluation science is also being shaped by global developments. Two major trends are relevant. First, the world is becoming effectively smaller, and national boundaries are dissolving. This is the result of the power of communication to break down the barriers that have separated the human race. The second global mega-trend is the growing strength of free market principles. Together, these two global developments mean that the factory worker in Detroit is in direct competition with counterparts in Stuttgart and Yokohama for economic prosperity. The economists tell us that ultimately global productivity will be optimized if this kind of competition is balanced and fair. True or not, there is no prospect that the reign of free market principles will be abolished any more than will the law of gravity itself. Regulatory scientists are involved in a global competition that is just as real for them as it is for auto workers. The competition is not confined to civil servants in Rockville, Uppsala, and London, but includes the expert physician in Lyon and the biostatistician in New Haven. The competition extends not only to authorities like the Medicines Control Agency (MCA) in the United Kingdom and the FDA, but also to academic and commercial organizations that can supply many of the same functions.

Within this global free market of drug regulatory approaches, there has been international cooperation. The International Conference on Harmonization of Technical Requirements for Licensing of Pharmaceuticals (ICH) has brought together the drug regulating authorities from Europe, Japan, and the United States. The objective is to achieve a single set of requirements in the areas of manufacturing, animal testing, and clinical trials that is acceptable to all three authorities for new drug approval. Much of the discussions in ICH have been based on science and therefore can be considered a discourse of drug regulatory science itself. ICH also has the valuable effects of highlighting the basic differences in approaches used by the three authorities and identifying those principles that appear to be universally utilized.

As an example, the European approach to new drug approval differs radically from that of the FDA. Europe's various national agencies, and more recently, the European Union authority, the Medicines Evaluation Agency, have small staffs of review professionals that rely heavily on outside academic consultants to produce what are called expert reports. These reports are the basis of approval decisions made by committees, also composed of outside experts. In contrast, the FDA is staffed with a large number of reviewing professionals who have training and experience within their assigned area of responsibility. While the FDA does utilize outside expertise through its advisory committees and occasionally in situations where internal expertise may require supplementation, the drug review process is performed and decisions are made by FDA scientists.

Probably the most fundamental difference between the European and FDA approaches is in the depth of review. In Europe, the expert reports are generally based on data summaries. At the FDA, raw data as well as data summaries submitted by the developer are independently checked, calculated, and analyzed by Agency reviewers. The FDA also has a comparatively rigorous field inspections system for examining an- 
imal and clinical investigations sites. The FDA, unlike European agencies, is also directly involved in reviewing and authorizing early human drug studies. In Europe, these phase I studies are generally supervised by local institutional ethics committees without direct regulatory authority involvement.

With fundamentally different approaches to drug regulation, how can there be a science of drug evaluation that undergirds the practices of both models? This seems more plausible when it is recognized that even though the regulatory authorities in Europe, Japan, and other countries do not perform some of the functions carried out by the FDA, these functions nonetheless are performed by other individuals or organizations, or by the drug developer itself. Even in the face of such different governmental approaches, it is certain that the evaluation scientists, whether privately or publicly supported, are, or should be, practicing the same science. The appropriate application of biostatistical methodology, the use of in vitro toxicology testing, and the design and interpretation of clinical studies are examples of scientific topics that have been or will be addressed within the ICH forum. It is likely that good review practice will become a subject of discussions that grow out of the ICH movement. This could mean not only that the requirements for drug developers are harmonized but also that practices of the evaluator/regulator are harmonized as well. The extent to which regulatory and evaluatory practices are harmonized will depend on the pace with which the drug evaluation science is developed and becomes widely recognized.

Widespread understanding and use of drug evaluation science will benefit drug discoverers and developers by making the goals and the processes involved clearer and the interactions with the regulators/evaluators more effective. Ultimately, everyone will benefit by the earlier availability of useful therapies. While art is required in any human endeavor, it is perhaps the involvement of too much art or personal discernment in the drug regulation process that has led some to perceive the FDA as inscrutable. "Ars brevis, scientia longa" 2 will allow professionals involved in the evaluation of therapies within and beyond the FDA to participate in the development of therapies in ways that best serve humankind.

\footnotetext{
${ }^{2}$ Less art, more science.
} 